\title{
Noise-induced quantum transport
}

\author{
Pulak Kumar Ghosh, Debashis Barik and Deb Shankar Ray* \\ Indian Association for the Cultivation of Science, Jadavpur, Kolkata 700 032, India
}

\begin{abstract}
We analyze the problem of directed quantum transport induced by external exponentially correlated telegraphic noise. In addition to quantum nature of the heat bath, nonlinearity of the periodic system potential brings in quantum contribution. We observe that quantization, in general, enhances classical current at low temperature, while the differences become insignificant at higher temperature. Interplay of quantum diffusion and quantum correction to system potential is analyzed for various ranges of temperature, correlation time and strength of external noise and asymmetry parameters. A possible experimental realization of the observed quantum effects in a superionic conductor placed in a random asymmetric dichotomous electric field has been suggested.
\end{abstract}

\footnotetext{
*Email Address: pcdsr@mahendra.iacs.res.in
} 


\section{INTRODUCTION}

Extracting useful work from unbiased noise has been a topical theme under 'ratchet effect' 1, 2, 3]. Because of its extraordinary success in explaining experimental observations on biochemical molecular motors active in muscle contractions [4, 5, 6, 7], observations on directed transport in photoreflective and photovoltic materials [8, 9], useful application in the separation of particles [10], theoretical issues on symmetry consideration, Brownian motion and second law of thermodynamics [11, 12, 13, 14], the problem has attracted wide attention in recent years. Although the ratchet effect can be achieved in a variety of ways the basic element of a typical Brownian ratchet essentially concerns the breaking of the detailed balance by an external periodic or fluctuating force applied to the Brownian particle moving in a periodic potential. This has been the subject a number of reviews and articles over the last decade. We refer to [1-3] for details.

While in the recent context of ratchet a major emphasis is laid on molecular pumps and motors in the realm of biophysics and chemistry, it would seem that a Brownian particle being a microscopic object [11], quantum effect is likely to make its presence felt in appropriate situation, particularly at low temperature. One thus expects the directed current or rectification of noise to be important in transport of quantum particles in quantum dots, wires related nanodevices [15, 16] and also in the context of superionic conductors 26, 27, 28]. Furthermore such studies are important also from the point of view of quantum-classical correspondence. Based on the quantum Langevin equation our aim here is to formulate a quantum ratchet problem where the external time dependent modulation that drives the quantum system out of equilibrium is a random telegraphic noise [17, 18, 19, 20]. To incorporate the elements of quantum theory in a ratchet device it is necessary to satisfy two basic requirements. First, quantization of classical motion must not break the symmetry of the ratchet device, or in other words, more specifically, quantization should not bring in any additional tilt to the potential or break its inversion symmetry or symmetry of the detailed balance. Second, the forcing must be unbiased, so that after appropriate averaging over ensemble or over the period of space or time no directional component should remain. Any approximation pertaining to the problem must conform to these requirements in any correct quantum formalism.

Keeping in view of the above considerations we first formulate the quantum stochastic 
dynamics of an overdamped particle and its approach to equilibrium. The quantum effects appear due to the nonlinearity of the potential and quantum noise of the heat bath. The introduction of the asymmetric telegraphic noise breaks the symmetry of the detailed balance to produce directed quantum transport. It is important to point out that the quantum effects observed by Linke et al [16] in their experimental work on quantum dot device are due to tunneling and wave reflection of electrons and this resulted in a reversal of current as predicted by Reimann[1, 15]. To explore the quantum effects discussed in this paper we first note that the quantum corrections have a different origin, e.g., the nonlinearity of the potential. A possible candidate for the experimental study of the present effect may be a superionic conductor, e.g., AgI. This system has been traditionally used [26, 27, 28] for measurement of current in presence of an external electric field directly or in terms of frequency dependent mobility. Typically in a superionic conductor like AgI, $I^{-}$ions form the lattice allowing the $\mathrm{Ag}^{+}$ions to move in a periodic potential of the form $\cos \frac{2 \pi x}{L}$, $\mathrm{L}$ being the lattice spacing. The lattice vibrations contribute to both the Langevin force as well as the frictional force on the $\mathrm{Ag}^{+}$ions maintaining the detailed balance at the thermal equilibrium. For slowly moving $\mathrm{Ag}^{+}$ions compared to lattice vibrations, white noise approximation is sufficient. An application of an external electric field at both ends of the conductor which fluctuates randomly between two values in an asymmetric way obeying the prescribed noise statistics, is expected to result in an observable current. In what follows from the present analysis is that apart from the low temperature contribution due to deep tunneling, the nonlinearity of the periodic pendulum potential $\cos \frac{2 \pi x}{L}$ (contribution beyond harmonic) contributes significantly to quantum corrections. The typical experimental parameters for the measurement of current in a superionic conductor has been given elsewhere [26]. It is also expected that quantum dots where the confining potential is truly periodic and nonlinear of similar type can offer themselves as good candidates for these studies.

The outlay of the paper is as follows: we first derive in Sec.II the basic equation describing quantum stochastic dynamics on a general footing followed by an overdamped description. Thermodynamic consistency has been stressed to avoid the pitfall of fictitious current generation. In Sec.III we show how an external dichotomous asymmetric noise can break the condition of detailed balance inducing a directed transport. Two limiting cases have been worked out in detail with a typically nonlinear periodic(cosine) potential as an example. The paper is concluded in Sce.IV. 


\section{A QUANTUM SYSTEM IN A SPATIALLY PERIODIC POTENTIAL AT EQUILIBRIUM}

\section{A. General aspects}

We consider a particle of mass $m$ moving in a periodic classical potential $V(x)$. The particle is coupled to a set of harmonic oscillators of unit mass acting as a bath. This is represented by the following system-reservoir Hamiltonian [21, 22, 23]

$$
\hat{H}=\frac{\hat{p}^{2}}{2 m}+V(\hat{x})+\sum_{j=1}^{N}\left\{\frac{\hat{p}_{j}^{2}}{2}+\frac{1}{2} \kappa_{j}\left(\hat{q}_{j}-\hat{x}\right)^{2}\right\}
$$

Here $\hat{x}$ and $\hat{p}$ are the coordinate and momentum operators of the particle and $\left\{\hat{q}_{j}, \hat{p}_{j}\right\}$ are the set of coordinate and momentum operators for the reservoir oscillators coupled linearly through the coupling constants $\kappa_{j}(j=1,2, \ldots)$. For the spatially periodic potential, we have $V(x)=V(x+L)$, where $L$ is the length of the period. The coordinate and momentum

operators follow the usual commutation rules $\{\hat{x}, \hat{p}\}=i \hbar$ and $\left\{\hat{q}_{i}, \hat{p}_{j}\right\}=i \hbar \delta_{i j}$. Eliminating the bath degrees of freedom in the usual way we obtain the operator Langevin equation for the particle

$$
m \ddot{\hat{x}}+\int_{0}^{\bar{t}} d \overline{t^{\prime}} \gamma\left(\bar{t}-\overline{t^{\prime}}\right) \dot{\hat{x}}\left(\overline{t^{\prime}}\right)+V^{\prime}(\hat{x})=\hat{\Gamma}(\bar{t})
$$

(Overdots refers to differentiation with respect to time $\bar{t}$ ) where noise operator $\hat{\Gamma}(\bar{t})$ and the memory kernel are given by

$$
\hat{\Gamma}(\bar{t})=\sum_{j}\left[\left\{\hat{q}_{j}(0)-\hat{x}(0)\right\} \kappa_{j} \cos \omega_{j} \bar{t}+\kappa_{j}^{1 / 2} \hat{p}_{j}(0) \sin \omega_{j} \bar{t}\right]
$$

and

$$
\gamma(\bar{t})=\sum_{j} \kappa_{j} \cos \omega_{j} \bar{t}
$$

respectively, with $\kappa_{j}=\omega_{j}^{2}$

Following Ref. [21-23] we then carry out a quantum mechanical average $\langle\ldots\rangle$ over the product separable bath modes with coherent states and the system mode with an arbitrary 
state at $\bar{t}=0$ in Eq.(2.2) to obtain a generalized quantum Langevin equation as

$$
m \ddot{\bar{x}}+\int_{0}^{\bar{t}} d \overline{t^{\prime}} \gamma\left(\bar{t}-\overline{t^{\prime}}\right) \dot{\bar{x}}\left(\overline{t^{\prime}}\right)+\overline{V^{\prime}}(\bar{x})=\bar{\Gamma}(\bar{t})+\bar{Q}\left(\bar{x}, \overline{\left\langle\delta \hat{x}^{n}\right\rangle}\right)
$$

where the quantum mechanical mean value of the position operator $\langle\hat{x}\rangle=\bar{x}$ and

$$
\bar{Q}\left(\bar{x}, \overline{\left.\left\langle\delta \hat{x}^{n}\right\rangle\right)}=\overline{V^{\prime}}(\bar{x})-\overline{\left\langle V^{\prime}(\hat{x})\right\rangle}\right.
$$

which by expressing $\hat{x}(\bar{t})=\bar{x}(\bar{t})+\delta \hat{x}(\bar{t})$ in $V(\hat{x})$ and using a Taylor series expansion around $\bar{x}$ may be rewritten as

$$
\bar{Q}\left(\bar{x}, \overline{\left\langle\delta \hat{x}^{n}\right\rangle}\right)=-\sum_{n \geq 2} \frac{1}{n !} \bar{V}^{n+1}(\bar{x}) \overline{\left\langle\delta \hat{x}^{n}\right\rangle}
$$

The above expansion implies that the nonzero anharmonic terms beyond $n \geq 2$ contain quantum dispersions $\overline{\left\langle\delta \hat{x}^{n}\right\rangle}$. Although we develop this section in general terms, we are specifically concerned here typically with periodic nonlinear potentials of the type $\sin \frac{2 \pi x}{L}$ or $\cos \frac{2 \pi x}{L}$ or their linear combinations and the like which have been used earlier in several contexts. The nonlinearity of the potential is an important source of quantum correction in addition to the quantum noise of the heat bath. The calculation of $\bar{Q}$ rests on the quantum correction terms $\overline{\left\langle\delta \hat{x}^{n}\right\rangle}$ which one determines by solving a set of quantum correction equations as given in the next section. Furthermore the c-number Langevin force is given by

$$
\bar{\Gamma}(\bar{t})=\sum_{j}\left[\left\langle\hat{q}_{j}(0)\right\rangle-\langle\hat{x}(0)\rangle \kappa_{j} \cos \omega_{j} \bar{t}+\kappa_{j}^{1 / 2} \hat{p}_{j}(0) \sin \omega_{j} \bar{t}\right]
$$

which must satisfy noise characteristics of the bath at equilibrium ,

$$
\begin{aligned}
\langle\bar{\Gamma}(\bar{t})\rangle_{S} & =0 \\
\left\langle\bar{\Gamma}(\bar{t}) \bar{\Gamma}\left(\overline{t^{\prime}}\right)\right\rangle_{S} & =\frac{1}{2} \sum_{j} \kappa_{j} \hbar \omega_{j}\left(\operatorname{coth} \frac{\hbar \omega_{j}}{2 k T}\right) \cos \omega_{j}\left(\bar{t}-\overline{t^{\prime}}\right)
\end{aligned}
$$

Eq.(2.10) expresses the quantum fluctuation-dissipation relation. The above conditions (2.9-2.10) can be fulfilled provided the initial shifted co-ordinates $\left\{\left\langle\hat{q}_{j}(0)\right\rangle-\langle\hat{x}(0)\rangle\right\}$ 
and momenta $\left\langle\hat{p}_{j}(0)\right\rangle$ of the bath oscillators are distributed according to the canonical thermal Wigner distribution [24, 25] of the form

$$
P_{j}\left(\left[\left\langle\hat{q}_{j}(0)\right\rangle-\langle\hat{x}(0)\rangle\right],\left\langle\hat{p}_{j}(0)\right\rangle\right)=N \exp \left\{-\frac{\frac{1}{2}\left\langle\hat{p}_{j}(0)\right\rangle^{2}+\frac{1}{2} \kappa_{j}\left[\left\langle\hat{q}_{j}(0)\right\rangle-\langle\hat{x}(0)\rangle\right]^{2}}{\hbar \omega_{j}\left[\bar{n}\left(\omega_{j}\right)+\frac{1}{2}\right]}\right\}
$$

so that the statistical averages $\langle\ldots\rangle_{s}$ over the quantum mechanical mean value $O$ of the bath variables are defined as

$$
\left\langle O_{j}\right\rangle_{s}=\int O_{j} P_{j} d\left\langle\hat{p}_{j}(0)\right\rangle d\left\{\left\langle\hat{q}_{j}(0)\right\rangle-\langle\hat{x}(0)\rangle\right\}
$$

Here $\bar{n}(\omega)$ is given by Bose-Einstein distributions $\left(e^{\frac{\hbar \omega}{k T}}-1\right)^{-1} . P_{j}$ is the exact solution of Wigner equation for harmonic oscillator [24, 25] and forms the basis for description of the quantum noise characteristics of the bath kept in thermal equilibrium at temperature $T$. In the continuum limit the fluctuation-dissipation relation (2.10) can be written as

$$
\left\langle\bar{\Gamma}(\bar{t}) \bar{\Gamma}\left(\overline{t^{\prime}}\right)\right\rangle=\frac{1}{2} \int_{0}^{\infty} d \omega \kappa(\omega) \rho(\omega) \hbar \omega \operatorname{coth}\left(\frac{\hbar \omega}{2 k T}\right) \cos \omega\left(\bar{t}-\overline{t^{\prime}}\right)
$$

where we have introduced the density of the modes $\rho(\omega)$. Since we are interested in the Markovian limit in the present context, we assume $\kappa(\omega) \rho(\omega)=\frac{2}{\pi} \gamma$, Eq.(2.13) then yields

$$
\left\langle\bar{\Gamma}(\bar{t}) \bar{\Gamma}\left(\overline{t^{\prime}}\right)\right\rangle=2 \bar{D}_{q} \delta\left(\bar{t}-\overline{t^{\prime}}\right)
$$

with

$$
\bar{D}_{q}=\frac{1}{2} \gamma \hbar \omega_{0} \operatorname{coth} \frac{\hbar \omega_{0}}{2 k T}
$$

$\omega_{0}$ refers to static frequency limit. Furthermore from Eq.(2.4) in the continuum limit we have

$$
\gamma\left(\bar{t}-\overline{t^{\prime}}\right)=\gamma \delta\left(\bar{t}-\overline{t^{\prime}}\right)
$$

$\gamma$ is the dissipation constant in the Markovian limit. In this limit Eq.(2.5) therefore reduces to

$$
m \ddot{\bar{x}}+\gamma \dot{\bar{x}}+\overline{V^{\prime}}(\bar{x})=\bar{\Gamma}(\bar{t})+\bar{Q}\left(\bar{x}, \overline{\left\langle\delta \hat{x}^{n}\right\rangle}\right)
$$


It is useful to work with dimensionless variables for the present problem to keep track of the relations between the scales of energy, length and time. The period $L$ of the periodic potential $V(x)$ determines in a natural way the characteristic length scale of the system. Therefore the position of the Brownian particle is scaled as

$$
x=\bar{x} / L
$$

Next we consider the timescales of the system. In absence of the potential and the noise term the velocity of the particle $\dot{x}(\bar{t}) \sim \exp \left(-\bar{t} / \tau_{L}\right)$ with $\tau_{L}=m / \gamma$, which represents the correlation time scale of the velocity the Brownian particle. To identify the next characteristic time $\tau_{0}$ we consider the deterministic overdamped motion due to the potential as $\gamma \frac{d \bar{x}}{d \bar{t}}=-\frac{d \bar{V}(\bar{x})}{d \bar{x}}$. Then $\tau_{0}$ is determined from $\gamma \frac{L}{\tau_{0}}=-\frac{\overline{\Delta V}}{L}$ as $\tau_{0}=\frac{\gamma L^{2}}{\Delta V}$ where $\overline{\Delta V}$ is the barrier height of the original potential. Hence time is scaled as $t=\frac{\bar{t}}{\tau_{0}}$. Furthermore the potential, the noise and the quantum correction terms are re-scaled as $V(x)=\bar{V}(\bar{x}) / \Delta \bar{V}$, $\Gamma(t)=\bar{\Gamma}(t) /(\Delta \bar{V} / L)$ and $\bar{Q} /(\Delta \bar{V} / L)$, respectively.

Hence dimensionless quantum Langevin equation reads as

$$
\mu^{*} \ddot{x}+\dot{x}=f(x)+\Gamma(t)
$$

Here over-dot(.) refers to differentiation with respect to scaled time $t$. Dimensionless $\operatorname{mass} \mu^{*}=\frac{m}{\gamma \tau_{0}}=\frac{\tau_{L}}{\tau_{0}}$ and

$$
f(x)=-V^{\prime}(x)+Q\left(x,\left\langle\delta \hat{x}^{n}\right\rangle\right)
$$

The noise properties of the quantum bath are then rewritten as

$$
\begin{gathered}
\langle\Gamma(t)\rangle_{s}=0 \\
\left\langle\Gamma(t) \Gamma\left(t^{\prime}\right)\right\rangle_{s}=2 D_{q} \delta\left(t-t^{\prime}\right)
\end{gathered}
$$

where

$$
D_{q}=\frac{\frac{1}{2} \hbar \omega_{0} \operatorname{coth} \frac{\hbar \omega_{0}}{2 k T}}{\overline{\Delta V}}
$$


The Fokker-Planck equation corresponding to Eq.(2.18) is given by

$$
\frac{\partial P(x, \dot{x}, t)}{\partial t}=\left[-\frac{\partial}{\partial x} \dot{x}+\frac{\partial}{\partial \dot{x}}\left(\frac{\dot{x}}{\mu^{*}}-\frac{f(x)}{\mu^{*}}\right)+\frac{D_{q}}{\mu^{* 2}} \frac{\partial^{2}}{\partial x^{2}}\right] P(x, \dot{x}, t)
$$

The above equation can be solved in the stationary state. The stationary probability density is

$$
P_{s t}(x, \dot{x})=N \exp \left[-\frac{\mu^{*} \dot{x}^{2}}{2}+\int_{0}^{x} \frac{f(y)}{D_{q}} d y\right]
$$

where $\mathrm{N}$ is the normalization constant which can obtained as

$$
\int_{-\infty}^{+\infty} d \dot{x} \int_{0}^{L} d x P_{s t}(x, \dot{x})=1
$$

It is easy to check that in the stationary state the mean velocity is equal to zero;

$$
\langle\dot{x}\rangle_{s}=\int_{-\infty}^{+\infty} \dot{x} d \dot{x} \int_{0}^{L} d x P_{s t}(x, \dot{x})
$$

Several points are now in order: (i) Eq.(2.23) suggests that the stationary distribution (2.21) is an equilibrium distribution because of the zero current condition. (ii) The equilibrium distribution Eq.(2.21) formally contains quantum corrections to all orders in $Q\left(x,\left\langle\delta \hat{x}^{n}\right\rangle\right)$. (iii) Since $Q\left(x,\left\langle\delta \hat{x}^{n}\right\rangle\right)$ essentially arises due to nonlinear part of the potential the nonlinearity and the quantum effects are entangled in this quantity modifying the classical part of the potential. Thus the classical force $-V^{\prime}(x)$ is modified by the quantum contribution. (iv) Since in the present scheme one may express the quantum mechanical operator $\hat{x}=x+\delta \hat{x}$ or $\dot{\hat{x}}=\dot{x}+\delta \dot{\hat{x}}$ where $x$ and $\dot{x}$ are quantum mechanical mean values and $\langle\delta \hat{x}\rangle=\langle\delta \dot{\hat{x}}\rangle=0$ by construction and $[\delta \hat{x}, \delta \dot{\hat{x}}]=i \hbar$ as noted earlier it follows that

$$
\langle\dot{\hat{x}}\rangle_{q s}=\langle\dot{x}+\delta \dot{\hat{x}}\rangle_{q s}=\langle\dot{x}\rangle_{s}+\left\langle\langle\delta \dot{\hat{x}}\rangle_{s}=\langle\dot{x}\rangle_{s}\right.
$$

The relation between three types of averages e.g., $\langle\ldots\rangle_{q s}$, quantum statistical; $\langle\ldots\rangle_{s}$ statistical average over quantum mechanical mean and $\langle\ldots\rangle$, quantum mechanical mean must be clearly distinguished. The relation Eq.(2.24) expresses the usual quantum current as a simple statistical average of the quantum mechanical mean value in the present c-number scheme and the decisive advantage of using this formalism is quite apparent. (v) In absence of quantum correction term $Q\left(x,\left\langle\delta \hat{x}^{n}\right\rangle\right)$ and $D_{q} \rightarrow \frac{\gamma k T}{\Delta V}$ as one approaches the classical limit 
$(k T \gg \hbar \omega)$, the quantum Langevin equation (2.18) reduces to classical Langevin equation.

(vi) The zero current situation or equivalently the equilibrium distribution function (2.21) ensures the condition of detailed balance in absence of any external driving. This condition is a necessity in the present context and the formalism since it guarantees that the quantum correction term does not give any tilt or bring any asymmetry on the classical periodic potential generating any unphysical current. This conclusion is also true for an overdamped situation which we deal in the next subsection.

\section{B. Equilibrium under overdamped condition}

Under overdamped condition the inertial term may be neglected and one obtains from Eq. (2.18)

$$
\dot{x}=f(x)+\Gamma(t)
$$

where over-dot (.) refers to differentiation with respect to dimensionless time $t$ defined

as $t=\frac{\bar{t}}{\tau_{0}}$ and $x=\frac{\bar{x}}{L}$. Therefore Eq.(2.25) gives the relation $\left\langle\frac{d \bar{x}}{d \bar{t}}\right\rangle_{s}=\frac{L}{\tau_{0}}\langle\dot{x}\rangle_{s}=v_{0}\langle f(x)\rangle_{s}$ or

$$
\left\langle\frac{d \bar{x}}{d \bar{t}}\right\rangle_{s}=v_{0} \int_{0}^{1} f(x) P_{s t}(x) d x
$$

Here we have denoted the characteristic velocity $v_{0}=L / \tau_{0}$. The equation for probability density function $P(x, t)$ corresponding to Eq.(2.25) is given by

$$
\frac{\partial P(x, t)}{\partial t}=-\frac{\partial J(x, t)}{\partial x}
$$

where the probability current

$$
J(x, t)=f(x) P(x, t)-D_{q} \frac{\partial P(x, t)}{\partial x}
$$

In the stationary state $P(x)=L t_{t \rightarrow \infty} P(x, t), \mathrm{J}$ is constant as

$$
J=f(x) P(x)-D_{q} \frac{\partial P(x)}{\partial x}
$$

The solution of above equation for $P(x)$ reads as

$$
P(x)=-\frac{J}{D_{q}} \exp [-\psi(x)] \int_{0}^{x} \exp [\psi(y)] d y+N \exp [-\psi(x)]
$$


where

$$
\psi(x)=-\int_{0}^{x} \frac{f(y)}{D_{q}} d y
$$

or $\psi(x)=\frac{V(x)-V(x)+\langle V(\hat{x})\rangle}{D_{q}}$ and $N$ is constant. The last relation follows from (2.6) and (2.19). Since $V(x)$ is periodic, i.e. $V(x)=V(x+1)$ we must have

$$
\psi(x)=\psi(x+1)
$$

For periodic boundary condition on (2.30) and from (2.31) it follows that,

$$
\frac{J}{D_{q}} \int_{x}^{x+1} \exp [\psi(y)] d y=0
$$

Since the above integral is non-zero an overdamped Langevin equation with periodic boundary condition shows $J=0$. This corresponds to an equilibrium situation with probability density function from (2.30),

$$
P(x)=N \exp [-\psi(x)]
$$

Normalization constant $\mathrm{N}$ is $\left[\int_{0}^{1} \exp [-\psi(x)]\right]^{-1}$. Therefore the quantum correction $Q\left(x,\left\langle\delta \hat{x}^{n}\right\rangle\right)$ in $\psi(x)$, as expected, can not break the detailed balance in the quantum system, nor the symmetry of the potential. This conclusion is an important check of the present formalism for a correct description of the equilibrium.

\section{EXTERNAL NOISE-INDUCED QUANTUM TRANSPORT}

Since at equilibrium detailed balance in the quantum stochastic system under overdamped condition forbids any transport we introduce an external noise on the system. The dynamics of the particle is described by the equation

$$
\dot{x}=f(x)+\Gamma(t)+\xi(t)
$$

where $\Gamma(t)$ is the quantum internal noise of the bath with the properties as noted earlier, $\xi(t)$ is a random telegraph noise also known as dichotomous noise, which takes two possible values $\xi(t)=\{-a, b\}$. If the probability of jumps per unit time from one state are given 
by $P(-a \rightarrow b)=\mu_{a}$ and $P(b \rightarrow-a)=\mu_{b}$ and if we assume $a \mu_{b}=b \mu_{a}$, then this external stochastic process can be described by the first two moments as

$$
\begin{gathered}
\langle\xi(t)\rangle=0 \\
\langle\xi(t) \xi(s)\rangle=\frac{Q_{I}}{\tau} \exp \left[-\frac{|t-s|}{\tau}\right]
\end{gathered}
$$

where the correlation time of the noise $\tau=\frac{1}{\mu_{a}+\mu_{b}}$ and the noise intensity $Q_{I}=\tau a b$, $\tau_{a}$ and $\tau_{b}$ are mean waiting times in the states $a$ and $b\left(\mu_{a}=\frac{1}{\tau_{a}}, \mu_{b}=\frac{1}{\tau_{b}}\right)$ respectively. Therefore the three parameters intensity $Q_{I}$, correlation time $\tau$, and asymmetry $\theta=b-a$ are the characteristics of the noise. For symmetrical noise $a=b$. The quantum nature of the problem therefore manifests itself in two ways; first, through quantum corrections in $f(x)$ which we consider in principle to all orders and secondly in quantum diffusion coefficient $D_{q}$ for the noise of the bath. The quantum equation of motion for joint probability densities can be mapped into a classical problem by defining

$$
P_{+}(x, t)=P(x, b, t) \quad ; \quad P_{-}(x, t)=P(x,-a, t)
$$

so that Fokker-Planck equation with jump processes are given by

$$
\begin{aligned}
& \frac{\partial P_{+}(x, t)}{\partial t}=-\frac{\partial}{\partial x}[f(x)+b] P_{+}(x, t)+D_{q} \frac{\partial^{2}}{\partial x^{2}} P_{+}(x, t)-\mu_{b} P_{+}(x, t)+\mu_{a} P_{-}(x, t) \\
& \frac{\partial P_{-}(x, t)}{\delta t}=-\frac{\partial}{\partial x}[f(x)-a] P_{-}(x, t)+D_{q} \frac{\partial^{2}}{\partial x^{2}} P_{-}(x, t)+\mu_{b} P_{+}(x, t)-\mu_{a} P_{-}(x, t)
\end{aligned}
$$

The total probability density $P(x, t)$ at any time is given by

$$
P(x, t)=P_{+}(x, t)+P_{-}(x, t)
$$

Eqs.(3.4) and (3.5) yield the equation of the motion for $P(x, t)$ as

$$
\frac{\partial P(x, t)}{\partial t}=-\frac{\partial}{\partial x}[f(x)] P(x, t)-\frac{\partial}{\partial x} W(x, t)+D_{q} \frac{\partial^{2}}{\partial x^{2}} P(x, t)
$$

where $W(x, t)$ is an auxiliary distribution function

$$
W(x, t)=b P_{+}(x, t)-a P_{-}(x, t)
$$


which follows the equation

$$
\frac{\partial W(x, t)}{\partial t}=-\frac{\partial}{\partial x}[f(x)+\theta] W(x, t)+D_{q} \frac{\partial^{2}}{\partial x^{2}} W(x, t)-\frac{1}{\tau} W(x, t)-a b \frac{\partial}{\partial x} P(x, t)
$$

The normalization conditions are

$$
\int_{c}^{c+1} P(x, t) d x=1 \quad ; \quad \int_{c}^{c+1} W(x, t) d x=0
$$

In the stationary state we obtain an expression for the constant current $J$ as

$$
-D_{q} P^{\prime}(x)+f(x) P(x)+W(x)=J
$$

and also we have

$$
\tau D_{q} W^{\prime \prime}(x)-\tau[\theta+f(x)] W^{\prime}(x)-\left[1+\tau f^{\prime}(x)\right] W(x)=Q_{I} P^{\prime}(x)
$$

$P(x)$ and $W(x)$ are the stationary solutions of the coupled equations (3.7) and (3.9). It is difficult to solve analytically the above two equations for arbitrary potential. In what follows we consider the solutions under two specific cases $(a)$ large correlation time and $(b)$ small correlation time.

\section{A. Large correlation time}

We return to Eqs.(3.11) and (3.12) and rewrite (3.12) as

$$
D_{q} \frac{d^{2} W(x)}{d x^{2}}-\frac{d}{d x}\{\theta+f(x)\} W(x)-\frac{1}{\tau} W(x)=a b \frac{d P(x)}{d x}
$$

For $\tau \gg 1$ we neglect the term with $\frac{1}{\tau}$. Integration over Eq. (3.13) then leads to

$$
D_{q} W^{\prime}(x)-[\theta+f(x)] W(x)=a b P(x)+D
$$

$D$ is constant. We now put the equilibrium solution for $P(x)$, Eq.(2.33) in (3.14) and solve it for $W(x)$ to obtain (we put $D=0$ to make the system free from bias due to external fluctuating force averaged over a period)

$$
W(x)=\exp \left[-\psi_{1}(x)\right]\left\{\frac{a b N}{D_{q}} \int_{0}^{x} \exp \psi_{2}(y) d y+C_{m}\right\}
$$




$$
\begin{gathered}
C_{m}=-\frac{a b N}{D_{q}} \frac{\int_{c_{0}}^{c_{0}+1} \exp \left[-\psi_{1}(x)\right] \int_{0}^{x} \exp \left[\psi_{2}(y)\right] d y d x}{\int_{c_{0}}^{c_{0}+1} \exp \left[-\psi_{1}(x)\right] d x} \\
\psi_{1}(x)=-\int_{0}^{x} \frac{\theta+f(y)}{D_{q}} d y \\
\psi_{2}(x)=-\int_{0}^{x} \frac{\theta}{D_{q}} d y \\
\psi(x)=-\int_{0}^{x} \frac{f(y)}{D_{q}} d y
\end{gathered}
$$

Here $N$ is given by normalization constant in (2.33). Putting the solutions for $W(x)$ and $P(x)$ (3.15 and 2.33) in (3.11) as a first approximation we obtain the expression for current as the lowest order iterative solution which is given by

$$
J=\frac{\int_{x}^{x+1} \exp \left[-\psi_{2}(y)\right]\left\{\frac{a b N}{D_{q}} \int_{0}^{y} \exp \left[\psi_{2}(z)\right] d z+C_{m}\right\} d y}{\int_{x}^{x+1} \exp [-\psi(y)] d y}
$$

The expression for current is valid for large correlation time of the dichotomous noise but formally takes into consideration of quantum effects to all orders. In order to check the consistency of the above expression we now examine the following limiting situations. First, we consider the dichotomous noise to be symmetric, i.e., $\theta=0$. $J$ then reduces to

$$
J=\frac{\int_{x}^{x+1}\left\{\frac{a b N}{D_{q}} y+C_{m}\right\} d y}{\int_{x}^{x+1} \exp [-\psi(y)] d y}
$$

and

$$
C_{m}=-\frac{a b N}{D_{q}} \frac{\int_{c_{0}}^{c_{0}+1} x \exp [-\psi(x)] d x}{\int_{c_{0}}^{c_{0}+1} \exp [-\psi(x)] d x}
$$

If now $V(x)$ is assumed to be of inversion symmetric, then $\langle V(\hat{x})\rangle$ is also symmetric and $C_{m}$ would be zero and $J=0$ in such situation since

$$
\begin{gathered}
\int_{-1 / 2}^{+1 / 2} x \exp [-\psi(x)] d x=0 \\
\int_{-1 / 2}^{+1 / 2} \frac{a b N}{D_{q}} x d x=0
\end{gathered}
$$

Therefore with symmetric potential and symmetric dichotomous noise, the current is zero even in the presence of quantum corrections. To obtain a quantum current it is necessary that 
either the periodic potential should be asymmetric and/or noise $\xi(t)$ should be asymmetric and vice-versa.

We now proceed to analyze the current under non-equilibrium condition and the related quantum effects. One of the prime quantities for this analysis is the potential $V(x)$ or the corresponding force term $f(x)$ given by

$$
f(x)=-\left[V^{\prime}(x)-Q\left(x,\left\langle\delta \hat{x}^{n}\right\rangle\right)\right]=-\frac{\partial}{\partial x}\left[V(x)+\sum_{n \geq 2} \frac{1}{n !} V^{n}(x)\left\langle\delta \hat{x}^{n}\right\rangle\right]
$$

The quantum correction terms can be determined as follows. We return to the operator equation (2.2) and put $\hat{x}(\bar{t})=\bar{x}(\bar{t})+\delta \hat{x}(\bar{t})$ and $\hat{p}(\bar{t})=\bar{p}(\bar{t})+\delta \hat{p}(\bar{t})$ where $\bar{x}(\bar{t})=\langle\hat{x}(\bar{t})\rangle$ and $\bar{p}(\bar{t})=\langle\hat{p}(\bar{t})\rangle$ are the quantum mechanical mean values of the operators $\hat{x}$ and $\hat{p}$ respectively. By construction $[\delta \hat{x}, \delta \hat{p}]=i \hbar$ and $\langle\delta \hat{x}\rangle=\langle\delta \hat{p}\rangle=0$. We then obtain the quantum correction equation

$$
m \delta \ddot{\hat{x}}+\int_{0}^{\bar{t}} d \bar{t}^{\prime} \gamma\left(\bar{t}-\bar{t}^{\prime}\right) \delta \dot{\hat{x}}\left(\bar{t}^{\prime}\right)+\bar{V}^{\prime \prime}(\bar{x}) \delta \hat{x}+\sum_{n \geq 2} \frac{1}{n !} \bar{V}^{n+1}(\bar{x})\left(\delta \hat{x}^{n}-\overline{\left\langle\delta \hat{x}^{n}\right\rangle}\right)=\hat{\Gamma}(\bar{t})-\bar{\Gamma}(\bar{t})
$$

Again in the overdamped limit we discard the inertial term $m \delta \ddot{\widehat{x}}$. We then perform a quantum mechanical average with initial product separable coherent states of the oscillators of the bath only to get rid of the internal noise term and to obtain the reduced operator equation for the system as

$$
\gamma \delta \dot{\hat{x}}+\bar{V}^{\prime \prime}(\bar{x}) \delta \hat{x}+\sum_{n \geq 2} \frac{1}{n !} \bar{V}^{n+1}(\bar{x})\left(\delta \hat{x}^{n}-\left\langle\overline{\left.\delta \hat{x}^{n}\right\rangle}\right)=0\right.
$$

With the help of (3.27) we then obtain the equations for $\overline{\left\langle\delta \hat{x}^{n}(t)\right\rangle}$

$$
\begin{gathered}
\frac{d}{d \bar{t}} \overline{\left\langle\delta \hat{x}^{2}\right\rangle}=\frac{1}{\gamma}\left[-2 \bar{V}^{\prime \prime}(\bar{x}) \overline{\left\langle\delta \hat{x}^{2}\right\rangle}-\bar{V}^{\prime \prime \prime}(\bar{x}) \overline{\left\langle\delta \hat{x}^{3}\right\rangle}\right] \\
\frac{d}{d \bar{t}} \overline{\left\langle\delta \hat{x}^{3}\right\rangle}=\frac{1}{\gamma}\left[-3 \bar{V}^{\prime \prime}(\bar{x}) \overline{\left\langle\delta \hat{x}^{3}\right\rangle}-\frac{3}{2} \bar{V}^{\prime \prime \prime}(\bar{x}) \overline{\left\langle\delta \hat{x}^{4}\right\rangle}+\frac{3}{2} \bar{V}^{\prime \prime \prime}(\bar{x}){\overline{\left\langle\delta \hat{x}^{2}\right\rangle}}^{2}\right]
\end{gathered}
$$

and so on. Taking into account of the lowest order contribution $\overline{\left\langle\delta \hat{x}^{2}\right\rangle}$ explicitly we may write

$$
d \overline{\left\langle\delta \hat{x}^{2}\right\rangle}=-\frac{2}{\gamma} \bar{V}^{\prime \prime}(\bar{x}) \overline{\left\langle\delta \hat{x}^{2}\right\rangle} d \bar{t}
$$


The overdamped deterministic motion gives $\gamma d \bar{x}=-\bar{V}^{\prime}(\bar{x}) d t$ which when used in (3.30) yields after integration

$$
\overline{\left\langle\delta \hat{x}^{2}\right\rangle}=\Delta_{q}\left[\bar{V}^{\prime}(\bar{x})\right]^{2}
$$

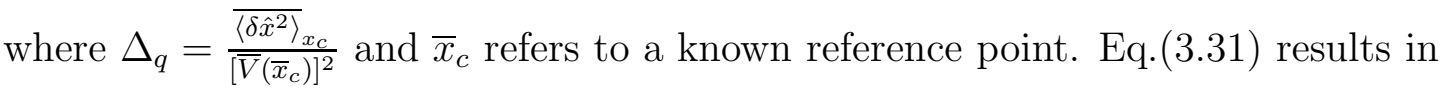

$$
\bar{f}(\bar{x})=-\left[\bar{V}^{\prime}(\bar{x})+\Delta_{q} \bar{V}^{\prime \prime \prime}(\bar{x})\left[\bar{V}^{\prime}(\bar{x})\right]^{2}\right]
$$

We now emphasize an important point. If the potential is symmetric, then the quantum correction in Eq. (3.32) is an odd function just as $V^{\prime}(x)$. This implies that quantum correction to classical potential has not destroyed the inversion symmetry of $V(x)$. Thus the approximation in deriving the leading order quantum effect is consistent with symmetry requirement of the problem.

To illustrate the nature of current we now consider a symmetric cosine potential with period $2 \pi$

$$
\bar{V}(\bar{x})=\frac{1}{2}(\cos \bar{x}+1)
$$

The force terms and other related quantities $\psi_{1}(x), \psi_{2}(x)$ and $\psi(x)$ after scaling, take the following forms:

$$
\begin{gathered}
f(x)=\pi\left[\sin 2 \pi x-\Delta_{q} \sin ^{3} 2 \pi x\right] \\
\psi_{1}(x)=-\left[\Delta_{1} \cos ^{3} 2 \pi x+\Delta_{2} \cos 2 \pi x+\frac{\theta}{D_{q}} x+\Delta_{3}\right] \\
\psi_{2}(x)=-\frac{\theta}{D_{q}} x \\
\psi(x)=-\left[\Delta_{1} \cos ^{3} 2 \pi x+\Delta_{2} \cos 2 \pi x+\Delta_{3}\right] \\
\Delta_{1}=-\frac{\Delta_{q}}{6 D_{q}} ; \quad \Delta_{2}=\frac{\Delta_{q}-1}{2 D_{q}} ; \quad \Delta_{3}=\frac{1-\frac{2}{3} \Delta_{q}}{2 D_{q}}
\end{gathered}
$$

In Fig.(1) we illustrate the variation of current for a fixed value of the system nonlinearity $\Delta_{q}(=0.04)$ and asymmetry parameter $\theta(=1.0)$ as a function of quantum diffusion 
coefficient $D_{q}$. One observes that with increase of $D_{q}$ the magnitude of current increases to a maximum followed by a decrease. For a fixed $D_{q}$ with increase of the strength of the external dichotomous noise (proportional to the product $a b$ ) the current increases. The effect of quantization of a classical ratchet is shown in Fig.(2), where we make a comparison of the current vs temperature profile for the classical and the quantum $\left(\Delta_{q}=0.3\right)$ cases for $a=1.75, b=2.75$. One observes that in the low temperature region the classical current is significantly lower in magnitude than the quantum current, and at high temperature the effect of quantization become insignificant.

\section{B. Short correlation time}

In the regime of short correlation time $\tau \ll 1$ of dichotomous noise we follow Kula et al [20] to expand $P(x), W(x)$ and $J$ in power series with $\tau$ as a smallness parameter;

$$
P(x)=\sum_{n=0}^{\infty} \tau^{n} P_{n}(x) \quad ; \quad W(x)=\sum_{n=0}^{\infty} \tau^{n} W_{n}(x) \quad \text { and } \quad J=\sum_{n=0}^{\infty} \tau^{n} J_{n}
$$

Making use of the above expressions in (3.11) and (3.12) we obtain the following set of equations,

$$
\begin{gathered}
-D_{q} P^{\prime}(x)+f(x) P_{n}(x)+W_{n}(x)=J_{n} \\
W_{n}(x)=D_{q} W_{n-1}^{\prime \prime}(x)-[\theta+f(x)] W_{n-1}^{\prime}(x)-f^{\prime}(x) W_{n-1}(x)-Q_{I} P_{n}^{\prime}(x)
\end{gathered}
$$

with $n=1,2,3 \ldots$

$$
W_{0}(x)=-Q_{I} P_{0}^{\prime}(x)
$$

The probability functions $P_{n}(x)$ obey the periodicity conditions and they are normalized over dimensionless period $(L=1)$. We thus obtain the zero order contributions as

$$
\begin{aligned}
J_{0} & =0 \\
P_{0}(x) & =N \exp \left[\int_{0}^{x} \frac{f(y)}{D_{q}+Q_{I}} d y\right]
\end{aligned}
$$


with normalization constant

$$
N^{-1}=\int_{0}^{1} \exp \left[\int_{0}^{x} \frac{f(y)}{D_{q}+Q_{I}} d y\right] d x
$$

The higher order contributions can be obtained following Kula et al. For the present purpose the leading order current is given by

$$
\begin{aligned}
J_{1} & =\left[\int_{0}^{1} N^{-1} P_{0}(x) d x \int_{0}^{1} N P_{0}^{-1}(x) d x\right]^{-1} \\
& \times\left[\frac{\theta Q_{I}}{\left(D_{q}+Q_{I}\right)^{2}} \int_{0}^{1} f^{2}(x) d x+\frac{Q_{I}^{2}}{\left(D_{q}+Q_{I}\right)^{3}} \int_{0}^{1} f^{3}(x) d x\right]
\end{aligned}
$$

The key quantity for the above equation is the force term $f(x)$ with leading order nonlinear correction (3.32). For the symmetric smooth cosine potential of the form $V(x)=\frac{1}{2}[\cos 2 \pi x+1]$ as given by (3.33),$f(x)$ is an odd function [Eq 3.34]. For asymmetric dichotomous fluctuations $(\theta \neq 0)$ and symmetric potential the leading order current is proportional to the integral $f^{2}(x)$; the integral over $f^{3}(x)$ being zero. On the other hand the current is proportional to the integral over $f^{3}(x)$ for $\operatorname{symmetric}(\theta=0)$ dichotomous noise. Therefore we have shown that in the short correlation time limit it is not possible to obtain any noise induced transport with symmetric noise and symmetric potential.

For $f(x)$ given by (3.34) one obtains explicitly the quantum current

$$
J_{1}=\frac{\pi^{2}}{2 I_{1} I_{2}} \frac{\theta Q_{I}}{\left(D_{q}+Q_{I}\right)^{2}}\left[\frac{5}{8} \Delta_{q}^{2}-\frac{3}{2} \Delta_{q}+1\right]
$$

where 


$$
\begin{aligned}
I_{1} & =\int_{0}^{1} \exp \left[-\int_{0}^{x} \frac{f(y)}{\left(D_{q}+Q_{I}\right)} d y\right] d x \\
I_{2} & =\int_{0}^{1} \exp \left[\int_{0}^{x} \frac{f(y)}{\left(D_{q}+Q_{I}\right)} d y\right] d x \\
\int_{0}^{x} \frac{f(y)}{\left(D_{q}+Q_{I}\right)} & =\Delta_{4} \cos ^{3} 2 \pi x+\Delta_{5} \cos 2 \pi x+\Delta_{6} \\
\Delta_{4} & =-\frac{\Delta_{q}}{6\left(D_{q}+Q_{I}\right)} ; \quad \Delta_{5}=\frac{\left(\Delta_{q}-1\right)}{2\left(D_{q}+Q_{I}\right)} \\
\Delta_{6} & =\frac{\left(1-\frac{2}{3} \Delta_{q}\right)}{2\left(D_{q}+Q_{I}\right)}
\end{aligned}
$$

We now numerically illustrate the behaviour of quantum current given by Eq.(3.46). The effect of quantization of the reservoir is apparent in Fig.(3) in the variation of current with $Q_{I}$ for several values of quantum diffusion coefficient $D_{q}$ of the heat bath for fixed $\Delta_{q}(=0.04), \theta(=1.0)$ and $\tau(=0.1)$. For small $D_{q}$, the current falls off monotonically after reaching maxima. The maxima and the current drops for higher values of quantum diffusion coefficient since thermalization prevails over the dynamics, in general. In Fig.(4) we compare the current vs temperature profile for the classical and the quantum $\left(\Delta_{q}=0.3\right)$ cases for fixed $a(=1.0), b(2.0)$ and $\tau(=0.1)$. We observe again that in the low temperature range the current is significantly higher for the quantum case.

In order to examine the influence of the correlation time $\tau$ of the nonthermal noise on current we plot in Fig.(5) the variation of current as a function of $D_{q}$ for several values of $\tau$ for fixed values of $a(=1.0), b(=2.0), \theta(=1.0)$ and $\Delta_{q}(=0.04)$. All the bell-shaped curves exhibit maxima at optimal $D_{q}$ values. Increase in correlation time $\tau$ results in enhancement of directed motion, and shift of the maxima, towards the origin. Physically this implies that departure from equilibrium is increasingly favored for larger correlation time of the external noise in this region.

The form for noise induced-current in the limit of short correlation time of the stochastic dichotomous process expresses the functional dependence of the current on $\tau, f, D_{q}$ and $Q_{I}$, through Eq.(3.46) as $J=\tau J_{1}$. It is interesting to comment on the effect of noise statistics of the stochastic processes on the current. This aspect has been explained carefully by Doering et al [18] sometime back in a classical context by considering a family of stochastic processes 
known as "Kangaroo processes" parameterized by an another probability distribution $p(z)$. Based on singular perturbation expansion in $\tau$ it has been shown that the expression for the current is modified by a prefactor which depends on the moments of the distribution $p(z) \quad\left(\left\langle z^{n}\right\rangle=\int z^{n} p(z) d z\right)$. It is apparent that consideration of such processes in the present quantum mechanical context is expected to give rise such a prefactor since the expression for current in the short correlation time limit in classical and quantum mechanical cases has a common structure.

\section{CONCLUSION}

Based on the traditional system-reservoir model we have formulated the quantum stochastic dynamics in the overdamped limit and analyzed the problem of a ratchet device with an external, exponentially correlated asymmetric dichotomous noise. The governing equations of motion are classical looking in form but quantum mechanical in their content. The quantization of the dynamics is manifested in two different ways. First, the harmonic oscillator reservoir is quantum mechanical in character and its internal noise characteristics and the fluctuation-dissipation relation are described by the canonical thermal Wigner distribution. The special advantage of using this c-number distribution (where width $D_{q}$ corresponds to the strength of fluctuation of the thermal bath) is that it remains a valid positive definite pure state distribution even at absolute zero. This allows the theory to be extended to the deep tunneling region which remains normally inaccessible in many theoretical treatments. Secondly, the nonlinearity of the potential brings in additional quantum contribution since the nonlinear terms of the potential beyond the harmonic one are entangled with quantum corrections. Therefore the system experiences an effective force term $f(x)$ comprising a classical $-V^{\prime}(x)$ plus a quantum correction term $Q\left(x,\left\langle\delta \hat{x}^{n}\right\rangle\right)$ as $f(x)=-V^{\prime}(x)+Q\left(x,\left\langle\delta \hat{x}^{n}\right\rangle\right)$ this consideration leads us to the form of generalized equilibrium distribution in terms of a nonlocal potential $\psi(x)$ as $P \sim \exp [\psi(x)]$ where $\psi(x)=\int_{0}^{x} \frac{f\left(x^{\prime}\right)}{D_{q}} d x^{\prime}$. The implication of this factor in ratchet effect or in Landauer blow-torch effect has been thoroughly examined [3, 14, 18, 20] by Van Kampen, Büttiker and others. A close look into the expression for current in Eq.(3.20) or Eq.(3.46) reveals that the origin of fluctuation induced current essentially rests on this factor and therefore the contribution of the nonlinearity induced quantum effect on this current becomes quite apparent. It is important to note that the es- 
sential requirements demanded by symmetry considerations and thermodynamic consistency condition have been fulfilled in our treatment. We now summarize the main conclusions of this study:

(i) We note that quantization can not break the symmetry of the ratchet device but, in general, may change the superposition of amplitudes of the periodic nonlinear function so that the current is significantly affected. This is apparent from the structure of the force term $f(x)$ pointed out earlier. For example, for a pendulum potential $V(x)=\frac{1}{2}(\cos x+1)$, which has been used for superionic conductors, $f(x)\left[=-V^{\prime}(x)-\Delta_{q} V^{\prime \prime \prime}(x)\left(V^{\prime}(x)\right)^{2}\right.$ for the leading order quantum correction where $\left.\Delta_{q} \sim O\left(h^{2}\right)\right]$ is a superposition of $\sin x$ and $\Delta_{q} \sin ^{3} x$, a classical and a quantum part, respectively. It therefore follows from the previous discussions that the quantum part of potential affects $\psi(x)$ and consequently the current.

(ii) Our formulation offers simple analytical solutions in two limiting cases, large and small correlation times of the telegraphic noise. We observe that while at low temperature quantization significantly enhances the classical current, at higher temperature the difference is insignificant. This may be interpreted in terms of an interplay between the quantum diffusion coefficient $D_{q}$ and the force term $f(x)$ appearing in the effective potential $\psi(x)$ as $\int_{0}^{x} \frac{f\left(x^{\prime}\right)}{D_{q}} d x^{\prime}$. As the temperature $T \rightarrow 0, D_{q}$ approaches to the value $\frac{1}{2} \hbar \omega_{0}$, the vacuum limit and also in the deep tunneling region the anharmonic terms in $f(x)$ do not contribute significantly, the integrand increases sharply. On the other hand, as the temperature increases, $D_{q}$ increases resulting in a decrease of $\psi(x)$. Since for the classical current, the quantum contribution to $f(x)$ is absent, one observes a crossover of the of the classical and the quantum current in an intermediate region of the temperature, where $D_{q}$ and $f(x)$ compete with each other and beyond which the quantum current is marginally lower than the classical current. For a further increase of temperature the classical and the quantum current merge identically, as expected.

(iii) We have examined the role of the strength of the external noise and the correlation time in the generation of noise-induced current. These factors, as usual are instrumental in moderating the statistical stationary state of the nonequilibrium system that lacks detailed balance. A possible experimental realization of the distinctive behaviour of a quantum ratchet in contrast to its classical counterpart in a superionic conductor driven by a dichotomous noisy electric field at low temperature has been suggested.

Our study is confined to analytical solutions in the two limits of correlation time of the 
external noise. A direct numerical simulation of quantum ratchet using c-number quantum Langevin equation with dichotomous noise over the entire range of correlation time is necessary to compliment these observations. Furthermore since the problem of quantum Brownian dynamics in a periodic (pendulum like) potential covers a wide area comprising Josephson super-current through a tunneling junction, Bloch-wall motion, Frochlich superconductors etc., we expect the formulation to be useful in a wider theoretical context. Application of the theory in related issues are also worth-pursuing and will be addressed elsewhere.

\section{Acknowledgments}

Thanks are due to the CSIR, Govt. of India, for a fellowship (PKG) and for partial financial support (grant No: 01/(1740)/02/EMR II).

[1] P. Reiman, Phys. Reps. 361, 57 (2002).

[2] F. Julicher, A. Ajdari and J. Prost, Rev. Mod. Phys. 69, 1269 (1997).

[3] M. O. Magnasco, Phys. Rev. Lett. 71, 1477 (1993); M. Büttiker, Z. Phys B 68, 161 (1987)

[4] E. H. Sepersu and T. Y. Tsons, J. Membr. Biol. 74, 191 (1983); J. Bio. Chem. 2591477 (1984)

[5] R. D. Vale and F. Oosawa, Adv. Biophys. 26, 97 (1990)

[6] J. Howard, Mechanics of Motor Proteins and the Cytoskeletons (Sinauer Associates, Sunderland 2001).

[7] T. Y. Tsong and R. D. Astumian, Bioelectrochems Bioenergy 211457 (1986); H.V. Westerhoff, T.Y. Tsong, P.B. Chock, Y.D. Chen, and R.D. Astumian, Proc. Natl. Acad. Sci. U.S.A. 83, 4734 (1986).

[8] A. M. Glass, D. von der Linde, T. J. Negran, Appl. phys. Lett. 25, 233 (1974).

[9] P. J. Sturman, Photovoltaic and Photo-refractive Effects in Noncentrosymmetric Materials (Gordon and Breach, Philadelphia 1992).

[10] J. Rousselet, L. Salome, A. Adjari and J. Prost, Nature 370, 446 (1994).

[11] C. Van den Broeck, R. Kawai, and P. Meurs, Phys. Rev. Lett. 93, 090601 (2004).

[12] H. S. Leff and A. F. Rex, Maxwell's Demon, Entropy, Information, Computing (Adam Hilger, 
Bristol 1990).

[13] K. Sekimoto, J.Phys. Soc. Jap 66, 1234 (1997).

[14] N. G. van Kampen, IBM J. Res. Develop. 32, 107 (1988).

[15] P. Reimann, M. Grifoni, and P. Hänggi, Phys. Rev. Lett. 79, 10 (1997).

[16] H. Linke, T. E. Humphrey, A. Lfgren, A. O. Sushkov, R. Newbury, R. P. Taylor, and P. Omling, Science 286, 2314 (1999).

[17] J. Łuczka, M. Niemiec and E. Piotrowski, J. Phys. A 26, 4849 (1993).

[18] C. R. Doering, W. Horsthemke and J. Riordan, Phys. Rev. Lett. 72, 2984 (1994).

[19] A. Mielke, Ann. Phys. 4, 476 (1995).

[20] J. Kula, T. Czernik and J. Łuczka, Phys. Rev. Lett. 80, 1377 (1998).

[21] D. Banerjee, B. C. Bag, S. K. Banik and D. S. Ray, Phys. Rev. E 65, 021109 (2002).

[22] D. Banerjee, B. C. Bag, S. K. Banik and D. S. Ray, J. Chem. Phys. 120, 8960 (2004).

[23] D. Barik and D. S. Ray, J. Chem. Phys. 121, 1681 (2004).

[24] E. P. Wigner, Phys. Rev. 40, 749 (1932).

[25] M. Hillery, R. F. O'Connell, M. O. Scully and E. P. Wigner, Phys. Reps. 106, 121 (1984).

[26] P. Brüesch, S. Strässler and H. R. Zeller, Phys. Status. Solidi. (a) 31, 217 (1975); P. Fulde, L. Pietronero, W. R. Schneider and S. Strässler, Phys. Rev. Lett. 35, 1776 (1975).

[27] T. Geisel, in Physics of Superionic Conductor, ed. by M. B. Soloman, Topic Current Phys., Vol. 15 (Springer, Berlin, Heidelberg, N. Y., 1997) p 201

[28] W. Dicterich, P. Fulde and I. Peschel, Adv. Phys. 29527 (1980) 


\section{Figure Captions}

Fig.1: Variation of current $(J)$ with quantum diffusion coefficient $D_{q}$ in the large correlation time limit for $\Delta_{q}=0.04, \theta=1.0$ and (i) $a=1.0$ and $b=2.0$ (solid line), (ii) $a=1.25$ and $b=2.25$ (dashed line), (iii) $a=1.5$ and $b=2.5$ (dot line) and (iv) $a=1.75$ and $b=2.75$ (dash-dot line).

Fig.2: Comparison of quantum and classical current $(J)$ vs temperature $(T)$ profile for the parameter set $a=1.75, b=2.75, \Delta_{q}=0.0$ (classical, dotted line) and $\Delta_{q}=0.3$ (quantum, solid line) in the large correlation time limit.

Fig.3: Variation of current $(J)$ with nonthermal noise strength $\left(Q_{I}\right)$ for different values of quantum diffusion coefficients $D_{q}=0.25$ (solid line), $D_{q}=1.5$ (dashed line), $D_{q}=3.0$ (dotted line) and $D_{q}=7.0$ (dash-dot line) for the parameter set $\Delta_{q}=0.04, \theta=1.0$ and $\tau=0.01$ in the short correlation time limit.

Fig.4: Comparison of quantum and classical current $(J)$ vs temperature $(T)$ profile for the parameter set $a=1.0, b=2.0, \tau=0.1$ and $\Delta_{q}=0.0$ (classical, dotted line) and $\Delta_{q}=0.3$ (quantum, solid line) in the short correlation time limit.

Fig.5: Variation of current $(J)$ with quantum diffusion coefficient for different value of correlation time $\tau$ of nonthermal noise $\tau=0.05$ (solid line), $\tau=0.07$ (dashed line) and $\tau=0.1$ (dotted line) for the parameter set $\Delta_{q}=0.04, \mathrm{a}=1.0, \mathrm{~b}=2.0$ and $\theta=1.0$ in the short correlation time limit. 


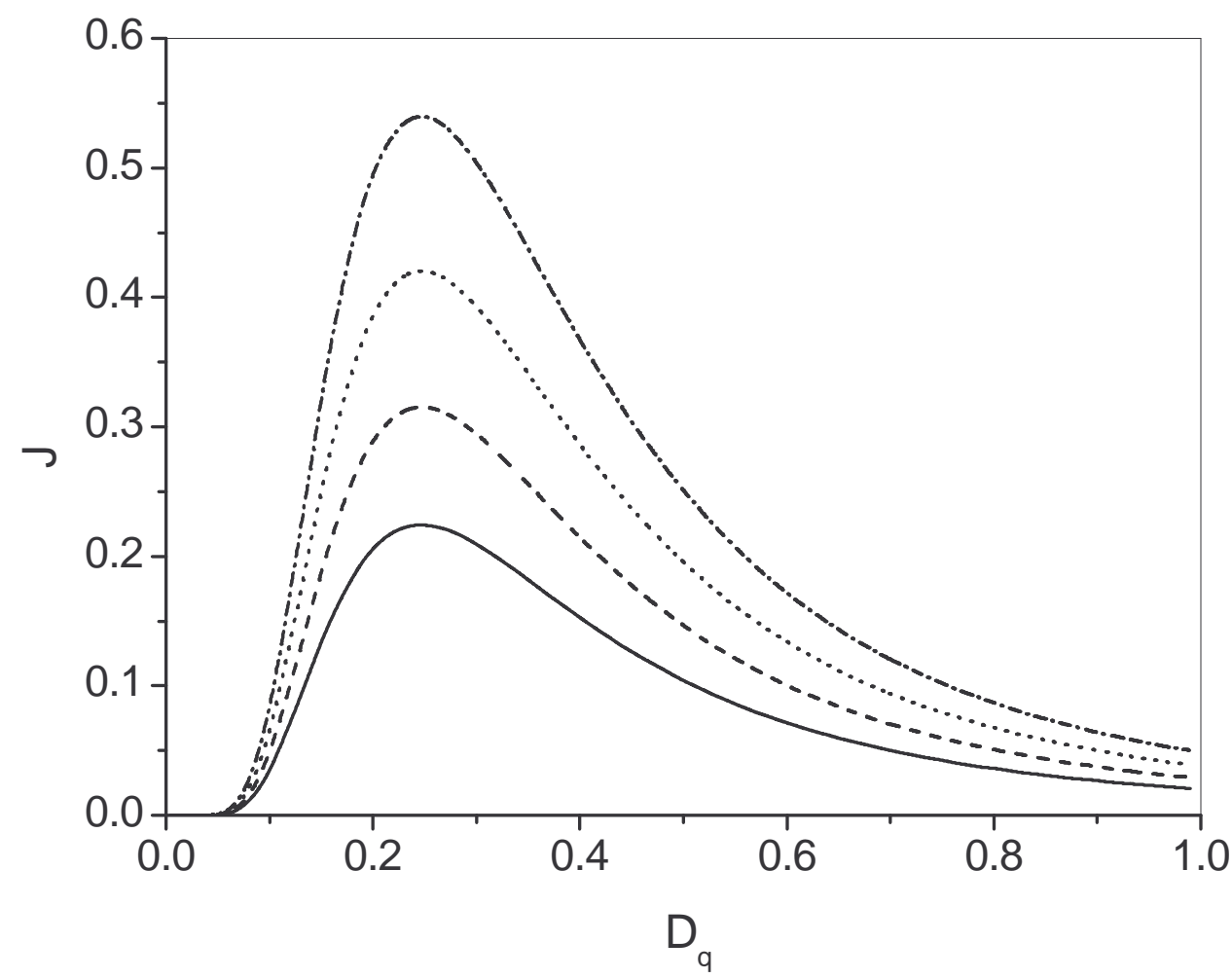

Fig. 1 


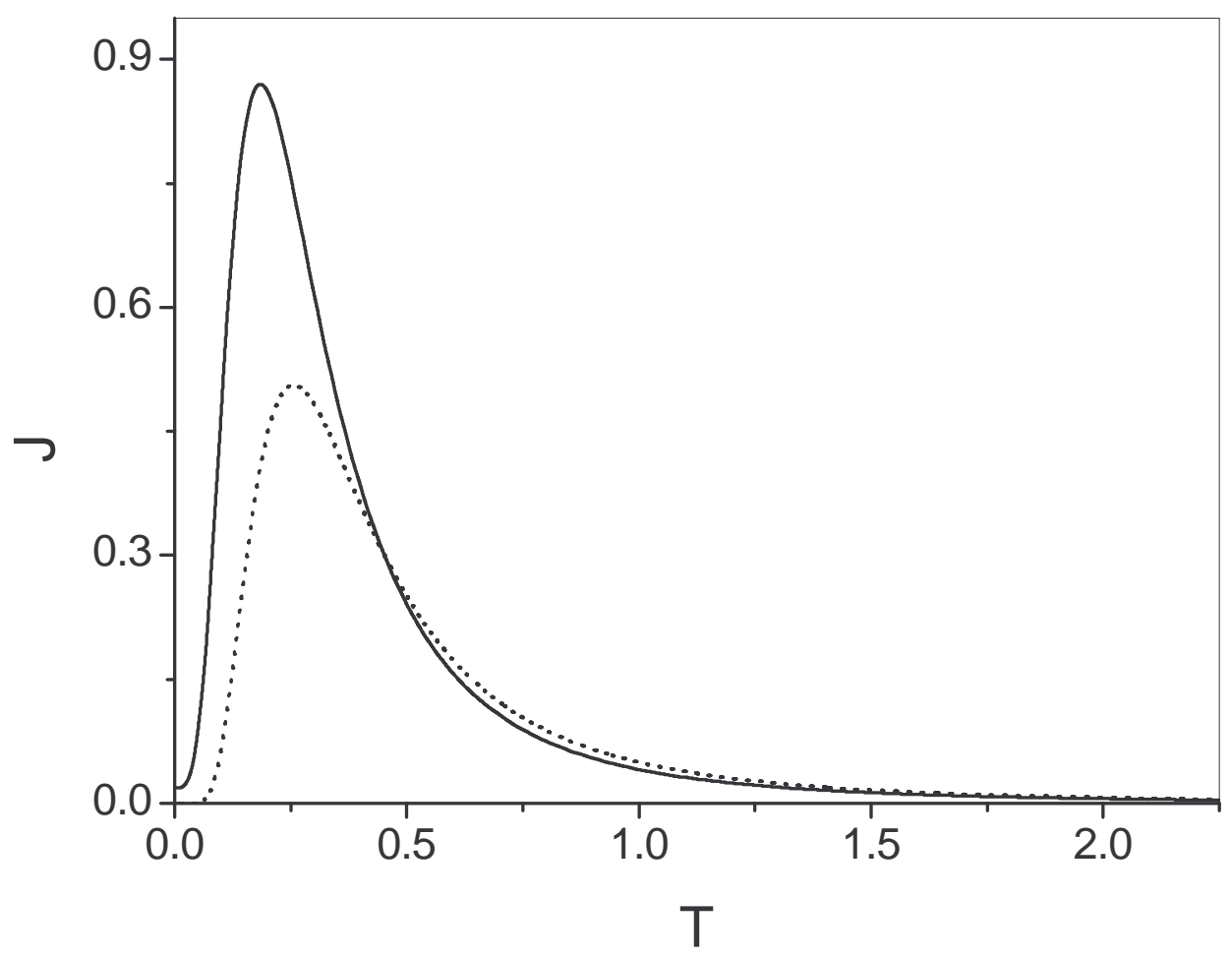

Fig.2 


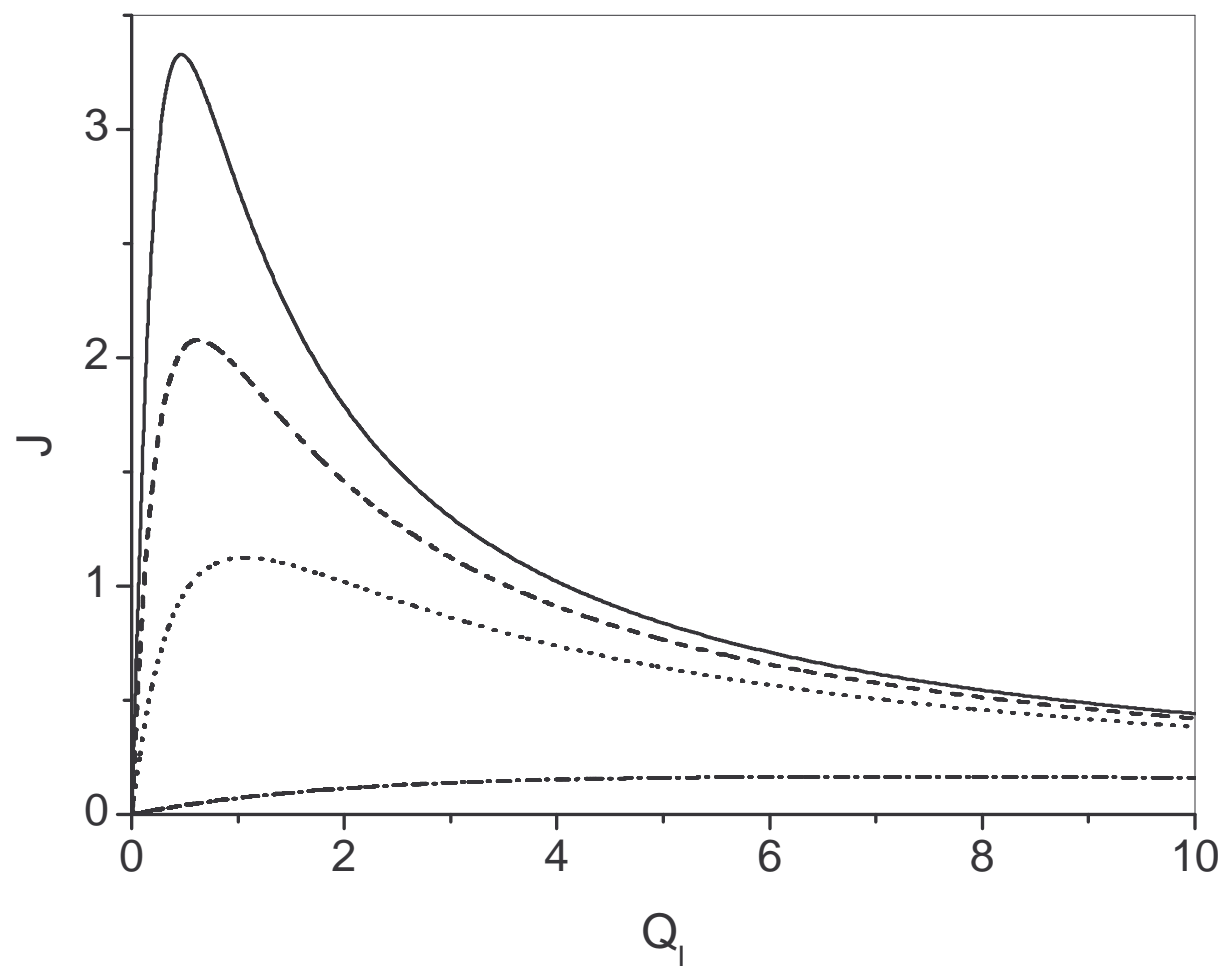

Fig.3 


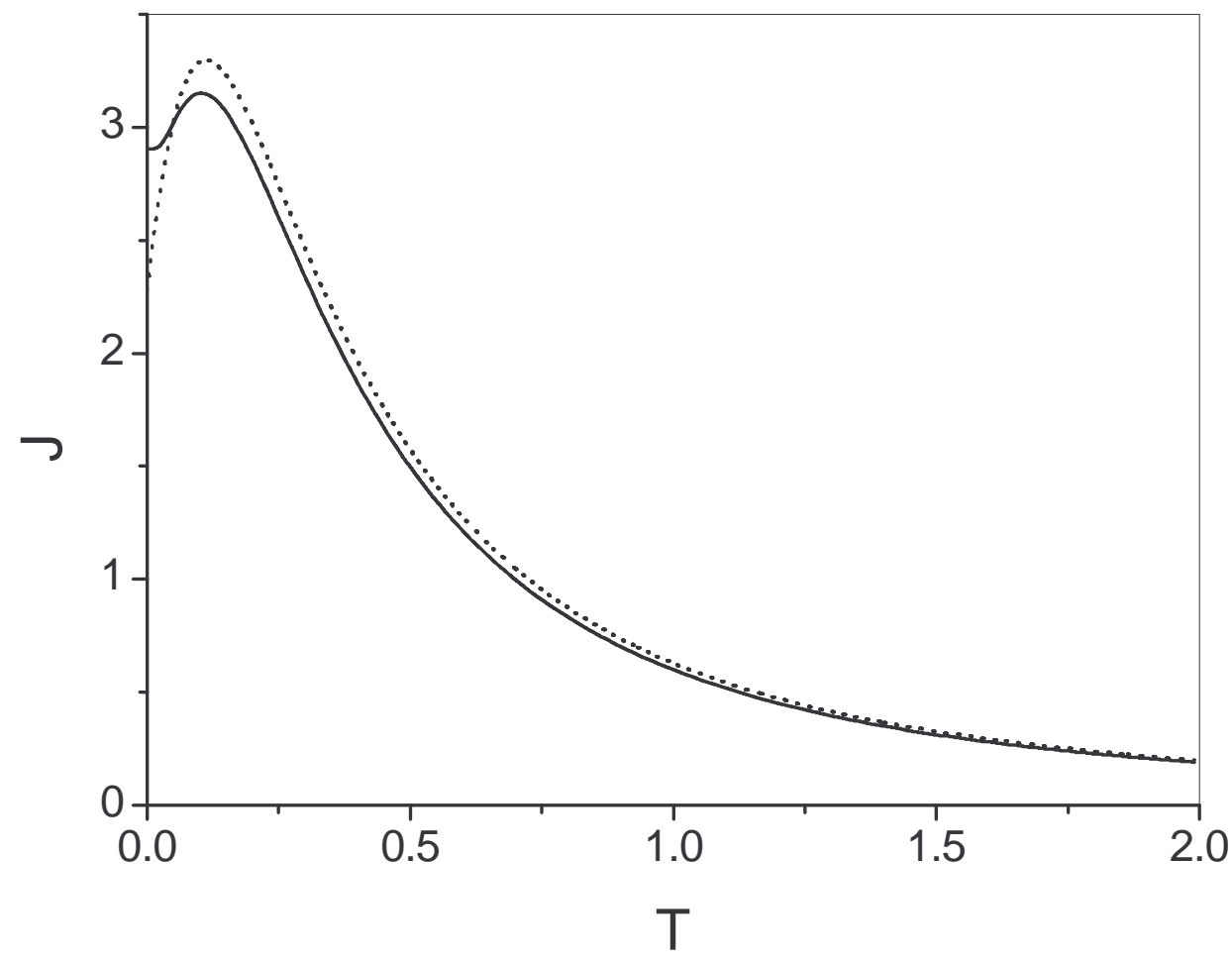

Fig.4 


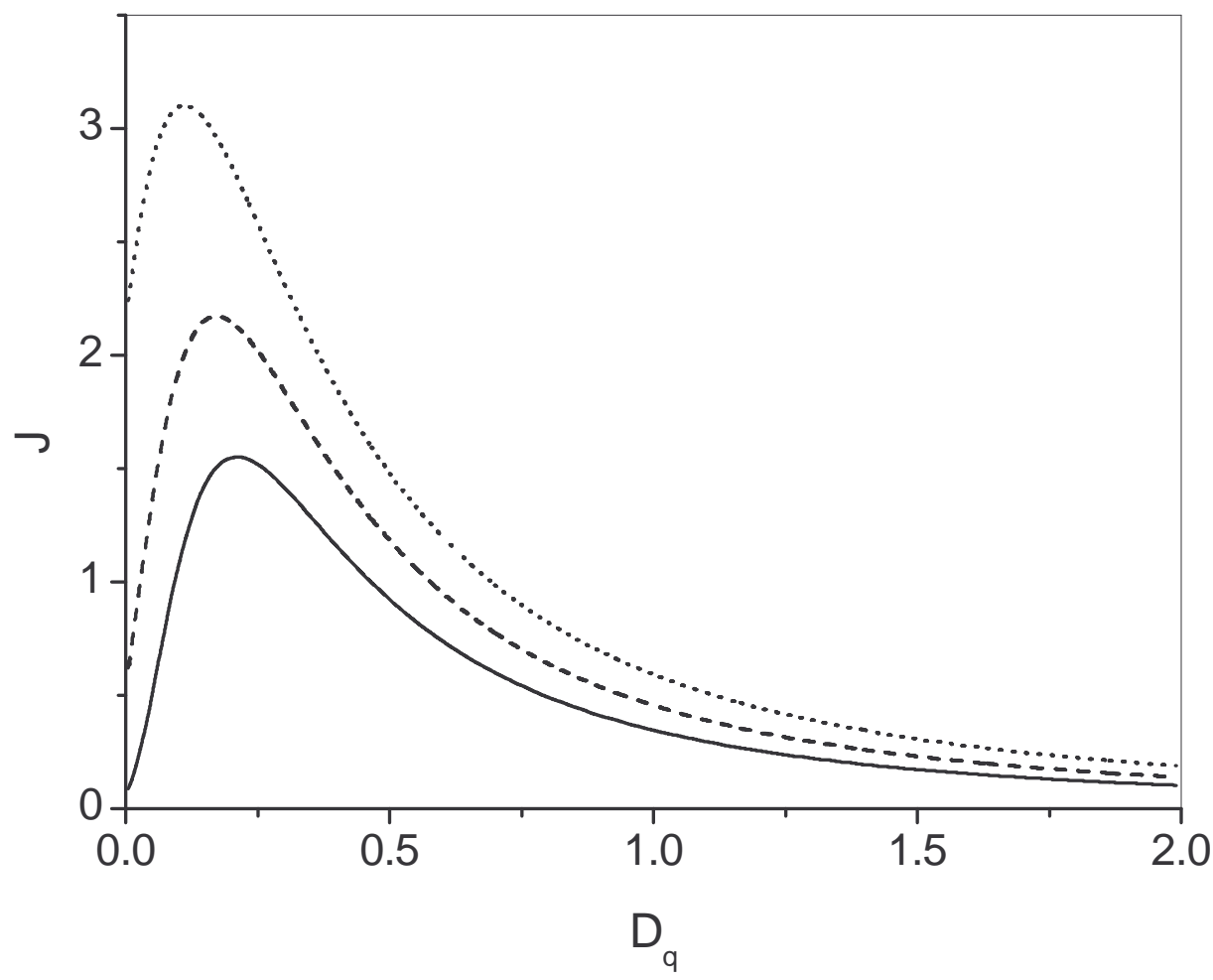

Fig.5 